\title{
POLARIZATION OBSERVABLES IN THE PHOTOPRODUCTION OF TWO PSEUDOSCALAR MESONS
}

\author{
W. ROBERTS ${ }^{1,2}$ \\ ${ }^{1}$ Department of Physics, Old Dominion University, Norfolk, VA 23529, USA \\ and \\ Continuous Electron Beam Accelerator Facility \\ 12000 Jefferson Avenue, Newport News, VA 23606, USA \\ 2 On leave at the Office of Nuclear Physics, Department of Energy \\ 19901 Germantown Road, Germantown, MD 20874.
}

\begin{abstract}
The many polarization observables that can be measured in process like $\gamma \mathrm{N} \rightarrow$ $\mathrm{M}_{1} \mathrm{M}_{2} \mathrm{~B}$, where $M_{1}$ and $M_{2}$ are pseudoscalar mesons and $B$ is a spin-1/2 baryon, are discussed. The relationships among these observables, their symmetries, as well as inequalities that they satisfy are briefly discussed. Within the context of a particular model for $\gamma \mathrm{N} \rightarrow \mathrm{NKK}$, some of the observables are calculated, and their sensitivity to the ingredients of the model, and hence to the underlying dynamics of the process, are discussed.
\end{abstract}

\section{Introduction and Motivation}

Polarization asymmetries are an essential ingredient in the interpretation of various meson production reactions in terms of the various resonances that contribute to the processes as real or virtual intermediate states. For instance, much of the information that we have on the light baryon resonances has been garnered from pion-nucleon and kaon-nucleon scattering experiments. In addition, photoproduction experiments have augmented the database of measurements that provide information on these resonances. The differential and total cross sections for these reactions, together with various polarization observables, are used to extract the amplitudes for the process, and these are then interpreted as arising from a number of resonant and non-resonant contributions ${ }^{1,2}$.

In this note, we briefly discuss a few aspects of polarization observables for processes in which two pseudoscalar mesons are photoproduced from a nucleon target. In the next section we briefly discuss the observables, the relationships among them, and the measurements needed for a 'complete' experiment. In section 3, we examine observables for $\gamma N \rightarrow K \bar{K} N$ in a 
model constructed using the phenomenological Lagrangian approach. In section 4, we present our conclusion and outlook.

\section{Observables in $\gamma N \rightarrow M_{1} M_{2} B$}

\subsection{Kinematics}

The center of mass kinematics for the process $\gamma N \rightarrow M_{1} M_{2} B$ are shown in fig. $1 . k$ is the four-momentum of the incident photon, $p$ that of the target nucleon and $p^{\prime}$ that of the recoil baryon. $\vec{k}$ is the three-momentum of the photon, which defines the $z$-axis, and the three-momenta $\vec{k}$ and $\vec{p}^{\prime}$ define the $x-z$ plane. $q_{i}$ is the four-momentum of meson $M_{i}$. The $z^{\prime \prime}$-axis is defined along the sum of the momenta $\vec{q}_{1}+\vec{q}_{2}$. The angles $\Theta$ and $\Phi$ define the momentum $\vec{q}_{1}$ in the $x^{\prime \prime} y^{\prime \prime} z^{\prime \prime}$ coordinate system (the $y$ - and $y^{\prime \prime}$-axes coincide).

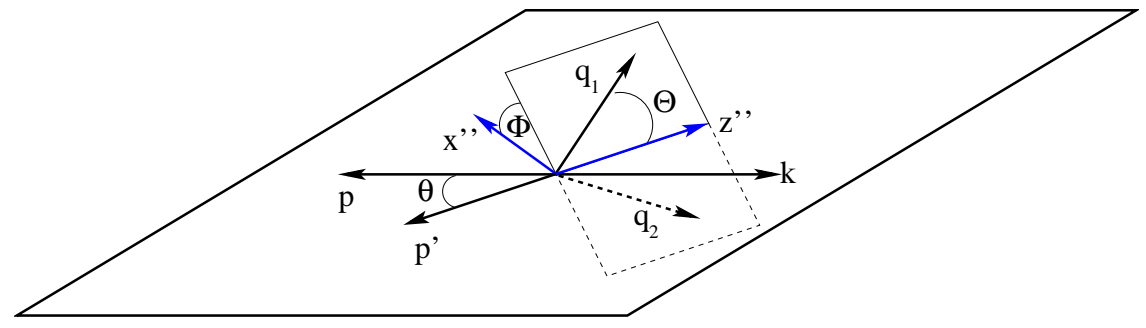

Figure 1. Kinematics for the proces $\gamma N \rightarrow M_{1} M_{2} B$. The variables are explained in the text.

\subsection{Reaction Rate and Relationships Among Observables}

The reaction rate $I$ for a process $\gamma N \rightarrow M_{1} M_{2} B$ can be written

$$
\begin{aligned}
\rho_{f} I= & I_{0}\left\{\left(1+\vec{\Lambda}_{i} \cdot \vec{P}+\vec{\sigma} \cdot \vec{P}^{\prime}+\Lambda_{i}^{\alpha} \sigma^{\beta^{\prime}} \mathcal{O}_{\alpha \beta^{\prime}}\right)\right. \\
& +\delta_{\odot}\left(I^{\odot}+\vec{\Lambda}_{i} \cdot \vec{P} \odot+\vec{\sigma} \cdot \vec{P} \odot{ }^{\odot}+\Lambda_{i}^{\alpha} \sigma^{\beta^{\prime}} \mathcal{O}_{\alpha \beta^{\prime}}^{\odot}\right) \\
& +\delta_{\ell}\left[\sin 2 \beta\left(I^{s}+\vec{\Lambda}_{i} \cdot \vec{P}^{s}+\vec{\sigma} \cdot \vec{P}^{s \prime}+\Lambda_{i}^{\alpha} \sigma^{\beta^{\prime}} \mathcal{O}_{\alpha \beta^{\prime}}^{s}\right)\right. \\
& \left.\left.+\cos 2 \beta\left(I^{c}+\vec{\Lambda}_{i} \cdot \vec{P}^{c}+\vec{\sigma} \cdot \vec{P}^{c \prime}+\Lambda_{i}^{\alpha} \sigma^{\beta^{\prime}} \mathcal{O}_{\alpha \beta^{\prime}}^{c}\right)\right]\right\},
\end{aligned}
$$

where $\vec{P}$ represents the polarization asymmetry that arises if the target nucleon is polarized, $\rho_{f}=\frac{1}{2}\left(1+\vec{\sigma} \cdot \vec{P}^{\prime}\right)$ is the density matrix of the recoiling nucleon, and $\mathcal{O}_{\alpha \beta^{\prime}}$ is the observable if both the target and recoil 
polarization are measured. The primes indicate that the recoil observables are measured with respect to a set of axes $x^{\prime}, y^{\prime}, z^{\prime}$, in which $z^{\prime}$ is along the direction of motion of the recoiling nucleon, and $y^{\prime}=y$ (the $z^{\prime}$-axis is opposite the $z^{\prime \prime}$-axis, and the $x^{\prime}$-axis is opposite the $x^{\prime \prime}$-axis). $\delta_{\odot}$ is the degree of circular polarization in the photon beam, while $\delta_{\ell}$ is the degree of linear polarization, with the direction of polarization being at an angle $\beta$ to the $x$-axis. The expressions for the observables in terms of the helicity or transversity amplitudes for the process are given in Ref. 3 .

There are 28 relations that arise among the 64 polarization observables of Eqn. (1) from consideration of the absolute magnitudes of the helicity or transversity amplitudes, and another 21 that arise from consideration of their phases, leaving 15 independent quantities. One example of such a relationship is

$$
\begin{aligned}
& {\left[P_{x^{\prime}}+\xi \mathcal{O}_{y x^{\prime}}+\zeta\left(P_{x^{\prime}}^{\odot}+\xi \mathcal{O}_{y x^{\prime}}^{\odot}\right)\right]^{2}+\left[P_{z^{\prime}}+\xi \mathcal{O}_{y z^{\prime}}+\zeta\left(P_{z^{\prime}}^{\odot}+\xi \mathcal{O}_{y z^{\prime}}^{\odot}\right)\right]^{2}} \\
& =\left[1+\xi P_{y}+\zeta\left(I^{\odot}+\xi P_{y}^{\odot}\right)\right]^{2}-\left[P_{y^{\prime}}+\xi \mathcal{O}_{y y^{\prime}}+\zeta\left(P_{y^{\prime}}^{\odot}+\xi \mathcal{O}_{y y^{\prime}}^{\odot}\right)\right]^{2} \cdot
\end{aligned}
$$

In this equation, $\xi$ and $\zeta$ can independently take either of the values \pm 1 , meaning that the single equation shown above actually represent four identities.

We can use the identities among the observables to write a number of inequalities that the polarization observables must satisfy. From eqn. (2), for example, we obtain

$$
\begin{array}{r}
\left|1+\xi P_{y}+\zeta\left(I^{\odot}+\xi P_{y}^{\odot}\right)\right| \geq\left\{\left|P_{y^{\prime}}+\xi \mathcal{O}_{y y^{\prime}}+\zeta\left(P_{y^{\prime}}^{\odot}+\xi \mathcal{O}_{y y^{\prime}}^{\odot}\right)\right|,\right. \\
\left.\left|P_{x^{\prime}}+\xi \mathcal{O}_{y x^{\prime}}+\zeta\left(P_{x^{\prime}}^{\odot}+\xi \mathcal{O}_{y x^{\prime}}^{\odot}\right)\right|, \quad\left|P_{z^{\prime}}+\xi \mathcal{O}_{y z^{\prime}}+\zeta\left(P_{z^{\prime}}^{\odot}+\xi \mathcal{O}_{y z^{\prime}}^{\odot}\right)\right|\right\}(3)
\end{array}
$$

where $\xi$ and $\zeta$ can again independently take either of the values \pm 1 . This inequality can be manipulated further to lead to

$$
\begin{array}{r}
1+P_{y}^{2}+\left(I^{\odot}\right)^{2}+\left(P_{y}^{\odot}\right)^{2} \geq\left\{P_{y^{\prime}}^{2}+\mathcal{O}_{y y^{\prime}}^{2}+\left(P_{y^{\prime}}^{\odot}\right)^{2}+\left(\mathcal{O}_{y y^{\prime}}^{\odot}\right)^{2},\right. \\
\left.P_{x^{\prime}}^{2}+\mathcal{O}_{y x^{\prime}}^{2}+\left(P_{x^{\prime}}^{\odot}\right)^{2}+\left(\mathcal{O}_{y x^{\prime}}^{\odot}\right)^{2}, \quad P_{z^{\prime}}^{2}+\mathcal{O}_{y z^{\prime}}^{2}+\left(P_{z^{\prime}}^{\odot}\right)^{2}+\left(\mathcal{O}_{y z^{\prime}}^{\odot}\right)^{2}\right\} .
\end{array}
$$

The full set of identities satisfied by the observables, as well as the various inequalities among them, are given in Ref. 3 .

\subsection{Required Measurements}

We can examine which observables need to be measured in order to extract the helicity or transversity amplitudes. As there are eight such ampli- 
tudes, a minimum of eight measurements must be made at each kinematic point (each observable depends on 5 kinematic variables) to obtain the absolute magnitudes of the helicity or transversity amplitudes. In terms of the transversity basis used in Ref. 3, the measurements needed are the differential cross section, along with $P_{y}, P_{y^{\prime}}, \mathcal{O}_{y y^{\prime}}, I^{\odot}, P_{y}^{\odot}, P_{y^{\prime}}^{\odot}$ and $\mathcal{O}_{y y^{\prime}}^{\odot}$.

The eight phases of the transversity amplitude mean that there are seven independent phase differences that can be extracted, and a minimum of seven measurements are needed for this. For instance, four of the relative phases require measurement of any four of the eight observables $P_{x^{\prime}}$, $P_{z^{\prime}}, \mathcal{O}_{y x^{\prime}}, \mathcal{O}_{y z^{\prime}}, P_{x^{\prime}}^{\odot}, P_{z^{\prime}}^{\odot}, \mathcal{O}_{y x^{\prime}}^{\odot}$ and $\mathcal{O}_{y z^{\prime}}^{\odot}$. Two more phases may then be extracted from measurement of any two observables from among $P_{x}, P_{z}$, $\mathcal{O}_{x y^{\prime}}, \mathcal{O}_{z y^{\prime}}, P_{x}^{\odot}, P_{z}^{\odot}, \mathcal{O}_{x y^{\prime}}^{\odot}$ and $\mathcal{O}_{z y^{\prime}}^{\odot}$. The remaining independent phase can then be extracted from one of the observables that arise from linearly polarized photons. A 'complete' set of experiments will therefore require measurement of single, double and triple polarization observables, using both linearly and circularly polarized photon beams, in addition to the differential cross section.

\subsection{Parity Invariance}

Unlike the case in which a single pseudoscalar meson is produced, the invocation of parity invariance does not reduce the number of independent helicity (or transversity) amplitudes needed to describe the process. The relationships that arise among the helicity amplitudes for $\gamma N \rightarrow M_{1} M_{2} B$ are

$$
\mathcal{M}_{-\lambda_{N}-\lambda_{N}^{\prime}}^{-\lambda_{\gamma}}(\theta, \Theta, \Phi)=(-1)^{\lambda_{\gamma}-\lambda_{N}+\lambda_{N}^{\prime}} \mathcal{M}_{\lambda_{N} \lambda_{N}^{\prime}}^{\lambda_{\gamma}}(\theta, \Theta, 2 \pi-\Phi) .
$$

These relations can not be used to decrease the number of independent helicity amplitudes, but they can be used to determine which observables are even or odd under the transformation $\Phi \leftrightarrow 2 \pi-\Phi$.

Examination of the full set of observables yields,

$$
\begin{array}{cccc}
I_{0}=-\mathcal{O}_{y y^{\prime}}^{c} & P_{y}=-P_{y^{\prime}}^{c} & P_{y^{\prime}}=-P_{y}^{c} & \mathcal{O}_{x x^{\prime}}=-\mathcal{O}_{z z^{\prime}}^{c} \\
\mathcal{O}_{x z^{\prime}}=\mathcal{O}_{z x^{\prime}}^{c} & \mathcal{O}_{y y^{\prime}}=-I^{c} & \mathcal{O}_{z x^{\prime}}=\mathcal{O}_{x z^{\prime}}^{c} & \mathcal{O}_{z z^{\prime}}=-\mathcal{O}_{x x^{\prime}}^{c} \\
P_{x}^{\odot}=\mathcal{O}_{z y^{\prime}}^{s} & P_{z}^{\odot}=-\mathcal{O}_{x y^{\prime}}^{s} & P_{x^{\prime}}^{\odot}=-\mathcal{O}_{y z^{\prime}}^{s} & P_{z^{\prime}}^{\odot}=\mathcal{O}_{y x^{\prime}}^{s} \\
P_{x}^{s}=-\mathcal{O}_{z y^{\prime}}^{\odot} & P_{z}^{s}=\mathcal{O}_{x y^{\prime}}^{\odot} & P_{x^{\prime}}^{s}=\mathcal{O}_{y z^{\prime}}^{\odot} & P_{z^{\prime}}^{s}=-\mathcal{O}_{y x^{\prime}}^{\odot}
\end{array}
$$

all at $\Phi=0, \Phi=\pi, \Phi=2 \pi$. All of the observables in Eqn. (6) are even under the transformation in $\Phi$. All other observables are odd under the transformation in $\Phi$, and vanish at $\Phi=0, \Phi=\pi$ and $\Phi=2 \pi$. 


\section{Model Calculation}

We now examine two aspects of the observables for a specific process, in a specific model, in an attempt to demonstrate their rich structure and their sensitivity to the underlying dynamics of the process. The process we treat is $\gamma N \rightarrow K \bar{K} N$, in a phenomenological Lagrangian approach discussed in detail in Ref. 4.

The polarization observables for this process are five-fold differential, which means that there are a number of different ways in which they can be displayed. Since it is not obvious how to display five-fold differential quantities, it is usual to integrate over some of the independent variables. In the following subsections, we integrate over some of the kinematic variables, showing the resulting observables as curves for different values of $\Phi$.

We note that since the observables are either even or odd under the transformation $\Phi \leftrightarrow 2 \pi-\Phi$, we do not integrate over this variable. In all the plots that follow, the observables are shown for four values of $\Phi$ : $\pi / 6, \pi / 4, \pi / 3$ and $\pi / 2$. We note that observables that are even in the $\Phi$ transformation could be displayed as Dalitz plots.

\subsection{Sub-threshold Resonance}

The $\Lambda(1405)$ resonance is one of the relatively well-established hyperons. It lies just below the $N K$ threshold, so its coupling (to $N K$ ) is not very well known. We examine the sensitivity of a number of observables to the presence of this state in the model calculation, and show the results for one observable, $\mathcal{O}_{y z^{\prime}}^{c}$, in fig. 2. The process process is $\gamma p \rightarrow n K^{+} \bar{K}^{0}$. In this figure, the results when the $\Lambda(1405)$ is excluded are shown in the left panel. The right panel shows the results we obtain when this state is included.

In this figure, the curves with the $\Lambda(1405)$ included are very different from those without it, especially near the lowest values of $m_{N \bar{K}}$. This feature serves to illustrate that in calculations such as this, 'small' contributions may not affect the cross section much, but can have significant effects in polarization observables.

\subsection{Pentaquark Search}

One very interesting question regarding the polarization observables is their possible sensitivity to exotic resonances, such as the $\Theta^{+}{ }^{5}$. If observables are found that show sensitivity to this state, they can be used to confirm its existence (or otherwise), assuming production mechanisms like those 

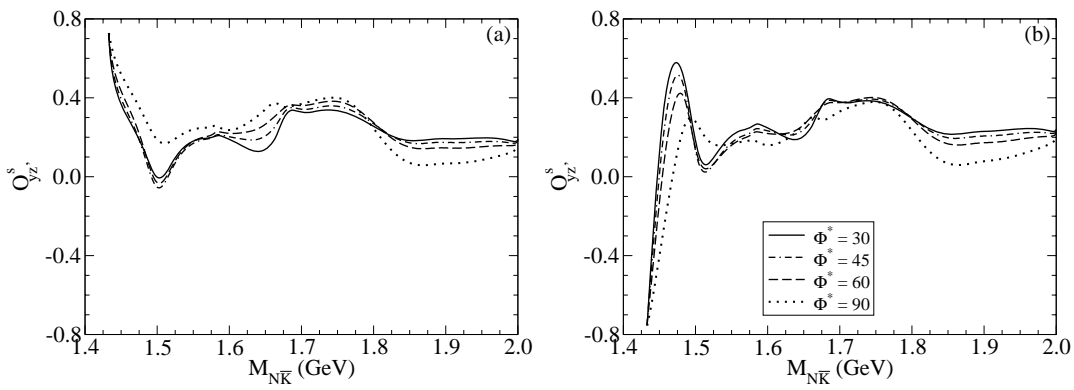

Figure 2. The observable $\mathcal{O}_{y z^{\prime}}^{c}$, showing its sensitivity to the sub-threshold resonance $\Lambda(1405)$. (a) results with the $\Lambda(1405)$ excluded in the calculation. (b) results when this state is included. All curves are shown as functions of $m_{N} \bar{K}$.

presented in Ref. 4. One of the disadvantages of using the differential cross section to search for states like this is that one state (or a few states) may provide a very large background against which a small signal must be sought. In the case of the pentaquark searches, the large backgrounds are provided by the $\Lambda(1520)$, along with other non-exotic hyperons, as well as mesons like the $\phi$. With polarization observables, large 'backgrounds' are not necessarily a problem, and the curves that we show illustrate what might be possible in pentaquark (or similar) searches.

In the model that we use for these calculations, the production cross section for the $\Theta^{+}$is of the order of a few nanobarns, consistent with the upper limit recently announced by researchers at $\mathrm{JLab}^{5}$. In the calculation, this size of cross section assumes that the pentaquark has $J^{P}=1 / 2^{+}$, and that mechanisms involving the $K^{*}$ are not important. In the same framework, the cross section obtained is significantly smaller if the state has $J^{P}=1 / 2^{-}$. In either case, the cross sections for producing the nonexotic hyperons, particularly the $\Lambda(1520)$ are several hundred times larger, and would contribute to the difficulty of extracting a $\Theta^{+}$signal, if the state were to exist.

Figs. 3 to $5\left(P_{x}^{\odot}, P_{z}^{\odot}\right.$ and $I^{\odot}$, respectively) show the curves that result when there is no $\Theta^{+}$in the calculation (the curves in (a)), and when a $\Theta^{+}$with $J^{P}=1 / 2^{+}$is included (the curves in (b)). In the case of Fig. 5 , the curves in (c) result when a $\Theta^{+}$with $J^{P}=1 / 2^{-}$is included in the calculation. In each case, the process is $\gamma p \rightarrow n K^{+} \bar{K}^{0}$. In fig. 5, the helicity asymmetry without the pentaquark is small, but recent work has demonstrated that even such a small observable is measurable with high precision at $\mathrm{JLab}^{6}$. When the pentaquark is included in the calculation, 

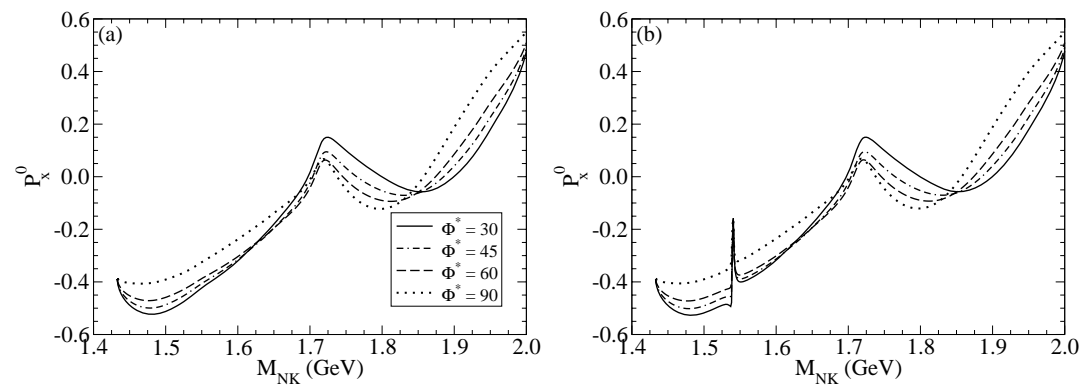

Figure 3. The observable $P_{x}^{\odot}$, showing its sensitivity to the exotic resonance $\Theta^{+}$. (a) results when the $\Theta^{+}$is excluded from the calculation. (b) results when a $\Theta^{+}$of positive parity is included in the calculation. All curves are shown as functions of $m_{N K}$.

this observable remains small, except for a structure in the region of the invariant mass of the pentaquark.
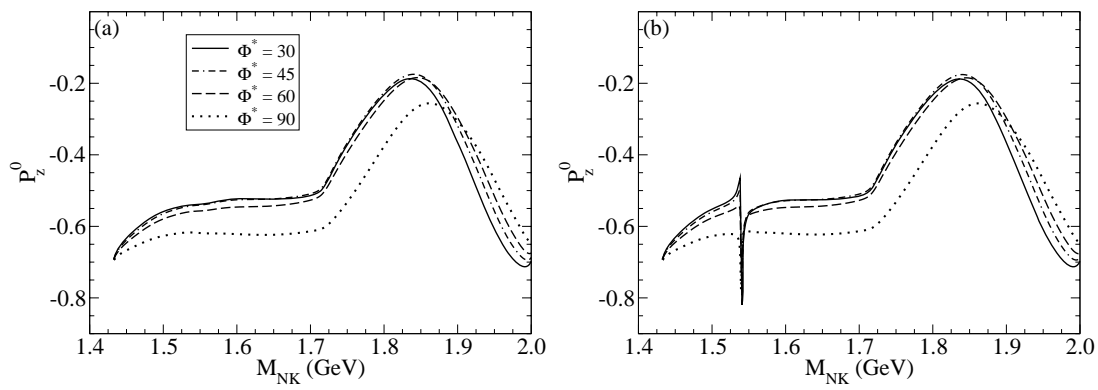

Figure 4. The observable $P_{z}^{\odot}$, showing its sensitivity to the exotic resonance $\Theta^{+}$. (a) results when the $\Theta^{+}$is excluded from the calculation. (b) results when a $\Theta^{+}$of positive parity is included in the calculation. All curves are shown as functions of $m_{N K}$.

For a pentaquark of positive parity, the signal in Fig. 5 is significant, but extraction could still be a challenge, as the width of the 'structure' is similar to the width of pentaquark (here, we are using a width of 1 $\mathrm{MeV}$ ). For a pentaquark with negative parity, the theoretical curves also show a significant structure, but it is somwehat less so than for the case of a positive parity pentaquark. One striking feature here is the difference in the 'sign' of the signal between the positive and negative parity cases, suggesting that this observable could act as an excellent parity filter for the pentaquark. Note that this asymmetry has already been measured at JLab for $\gamma p \rightarrow p \pi^{+} \pi^{-6}$. Thus, it may be possible to measure it for $\gamma N \rightarrow N K \bar{K}$ 
relatively quickly.
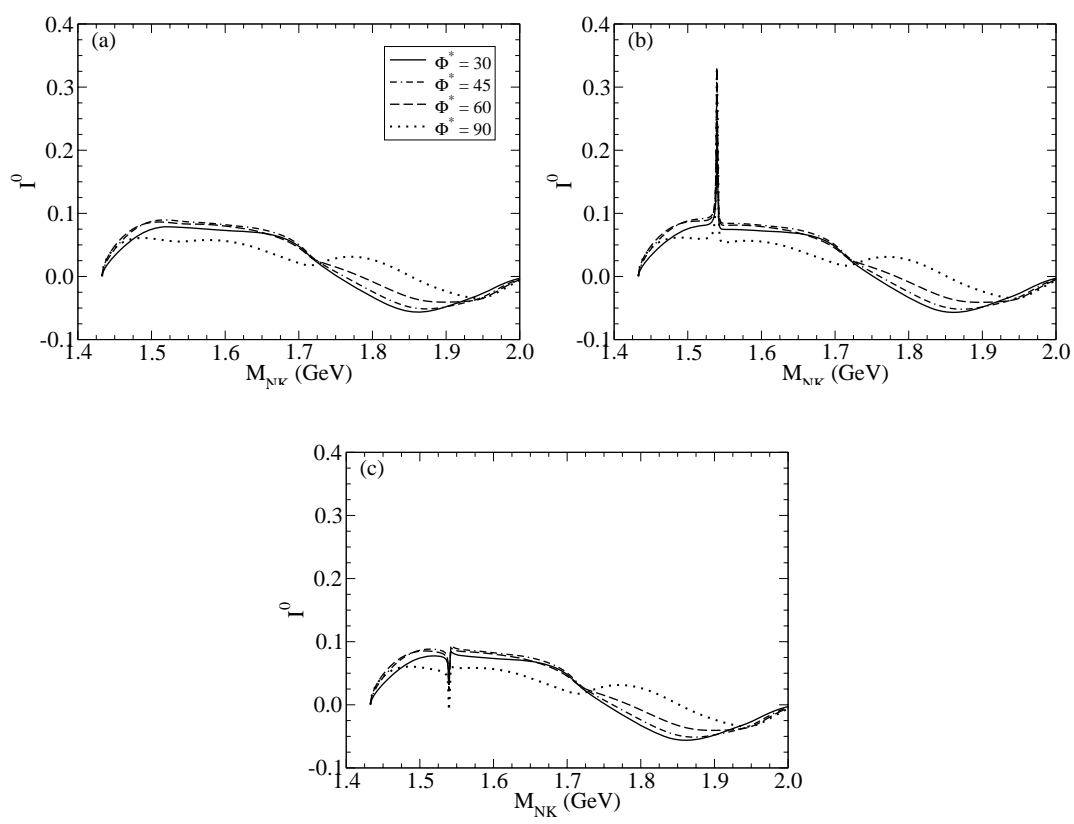

Figure 5. The beam asymmetry, $I^{\odot}$, showing its sensitivity to the exotic resonance $\Theta^{+}$. (a) results when the $\Theta^{+}$is excluded from the calculation. (b) results when a $\Theta^{+}$ of positive parity is included in the calculation and (c) results when a $\Theta^{+}$of negative parity is included in the calculation. All curves are shown as functions of $m_{N K}$.

Figs. 3 and 4 show similar structures in the curves for $P_{x}^{\odot}$ and $P_{z}^{\odot}$. Note that in all cases, the structures stand out clearly for two reasons. The first is that the pentaquark is a narrow state, and the 'width' of any structure that might be observed will be similar to that of the state giving rise to the structure. The second reason is that the $\Theta^{+}$is the only resonance in the $n K^{+}$channel. All other resonances are in the $n \bar{K}^{0}$ channel. The kinematic reflections from these resonances will show up, as can be seen in figs 3 and 4 , but the presence of the $\Theta^{+}$in this channel has a marked effect. Note that in fig. 4, this observable is predicted to be large (in the framework of the model used) and negative. A number of other observables utilizing linearly polarized photons (not shown here) show similar strctures for the pentaquark.

It must be emphasized here that the potential signals shown above arise in the full model, including the contribution of the $\Lambda(1520)$. In the model, 
the cross section for production of this state is several hundred times larger than the cross section for producing the pentaquark. Despite this inconvenient' ratio of production cross sections, the polarization observables explored above give clear signals for the pentaquark, and extraction of these signals will depend mainly on the energy resolution possible in any experiment, and much less so on the necessity of extracting a small signal from a large background. It must also be noted that the 'heights' of the signals shown for the pentaquark are independent of its width: only the width of the signals reflect the width of the pentaquark in the framework of the model used.

\section{Conclusion and Outlook}

The results presented above were obtained in the context of a particular model, and as such, they are clearly model dependent. Nevertheless, within the framework of this particular model, we have attempted to show how useful these polarization observables can be by exploring their sensitivity to a few details of the model. We are unable to comment on what other models would predict for such observables. In the case of the signals shown for the pentaquark, it would be unwise for us to speculate on the nature of such signals in other models.

We have attempted to convey a number of points about the polarization observables developed in Ref. 3. The first point is that, however they are displayed, these observables exhibit an enormously rich structure, reflecting the degree of complexity in the underlying dynamics. This sensitivity to the various contributions leading to the final state being studied, especially to 'small' contributions, provides an indispenable tool that will need to be fully exploited in our attempts to understand processes like the ones discussed herein. Such processes are expected to be among the primary sources of information in the on-going attempts to understand the dynamics of soft QCD.

A number of these observables should be accessible in the near future at existing facilities, in a number of different processes. The obvious applications are to the process discussed herein, $\gamma N \rightarrow N K \bar{K}$, and to $\gamma N \rightarrow N \pi \pi$. However, final states like $N \eta \pi, N \eta \eta, Y K \pi$ (where $Y$ is a $\Lambda$ or $\Sigma$ ), $Y K \eta$, and even $K K \Xi$, will require the same kinds of measurements in order to disentangle the various contributions leading to them. In the processes that produce hyperons in the final states, their various self-analysing decays provide access to recoil polarization measurements, thus opening up 
more possibilities. Many of these opportunities will have to be seized for continued progress to be made in our understanding of baryon spectroscopy.

\section{Acknowledgements}

This work was supported by the Department of Energy through contract DE-AC05-84ER40150, under which the Southeastern Universities Research Association (SURA) operates the Thomas Jefferson National Accelerator Facility (TJNAF).

\section{References}

1. See, for example, B. H. Bransden and R. G. Moorhouse, The Pion Nucleon System, Princeton University Press, New Jersey, 1973.

2. See, for example, J. S. Hyslop, R. A. Arndt, D. Roper and R. L. Workman, Phys. Rev. D46, 961 (1992);

B. R. Martin, Nucl. Phys. B94, 413 (1975).

3. W. Roberts and T. Oed, Phys. Rev. C71, 055201 (2005).

4. W. Roberts, Phys. Rev. C70, 065201 (2004).

5. See for example, V. Burkert, arXiv:hep-ex/0510309, and references therein.

6. S. Strauch et al. [CLAS Collaboration], Phys. Rev. Lett. 95162003 (2005). 